\title{
THE ESTIMATION OF WESTWARD DRIFT IN MGF ON THE NORTH AMERICA AND THE EUROPE
}

\author{
G.Bayanjargal \\ Research Center for Astronomy and Geophysics, \\ Mongolian Academy of Sciences (MAS), Ulaanbaatar, Mongolia \\ Email:gbayan27@yahoo.com
}

\begin{abstract}
In this paper, we have studied the westward drift in secular variation of MGF by the dynamo theory and the observatories' data of intermagnet.org on the North America and the Europe (1991-2006).

The longitudinal $\omega=-0.247^{\circ} / y r$ and latitudinal $\dot{\theta}=0.179 \%$ rr drifts exist on the North America. And longitudinal $\omega=-0.240^{\circ} / y r$ drift is also defined on the Europe in (1991-2006). These drifts are approximation with other investigation's results.
\end{abstract}

Keywords: Dynamo theory for mean geomagnetic field, westward drift, secular variation.

\section{Introduction}

Westward drift in secular variation of geomagnetic field has been studied since the 16 th century. We write briefly from prior investigations' results: Bauer studied westerly drift of the line zero declination tracing. He obtained the mean velocity of $\omega=0.22^{\circ} / y r$ and its direction back to the middle sixteenth sentry. Bullard and others studied the non-dipole field at epoch 1907.5 and 1945. They determined that the drift velocity is $\omega=0.18^{\circ} / y r$ [3]. T.Yukutake estimated that value of westward drift is $\omega=0.20^{\circ} / \mathrm{yr}$ [1]. Zigang Wei et all estimated that value of westward drift is $\omega=0.18^{\circ} / y r$ by the research during (19002000) [4]. These researches are based mainly on the method of sphere harmonic potential for the geomagnetic field.

If we have got the geomagnetic field's distribution function of space and time, can we define the westward drift on the Earth's little area such as a continent or any part of continents by other method from the above method? Is there any theoretical eventuality to decide this problem? Our purpose is to answer these questions. In this paper, we used the geomag- netic field's distribution function of space and time on the North America and Europe. These distribution functions were defined above article named the distribution function of MGF on space and time on the North America and the Europe.

The mean field dynamo theory for earth's liquid core

Strong toroidal field is sourced in subshell in the Earth's inner core by J.Marven Herndon's postulate for the main geomagnetic field[7],[9],[8]. And there are irregular fluid motions in the outer liquid core by dynamo theory [1]. The interaction between the motions and toroidal field would produce irregular magnetic fields with small scale. The irregular magnetic fields are called non-dipole part of the main geomagnetic field (MGF).

Variation with long period exists in the non-dipole part of the MGF. The variation is called secular variation.

On the surface of Earth, the westward drift in secular variation of the MGF is also observed by the causing of the motions in the liquid core.

The governing differential equation for 
the dynamic of the geomagnetic field is written on the $\mathrm{Eq}(1)$. The $\mathrm{Eq}(1)$ is named the induction equation.

$$
\frac{d \vec{B}}{d t}=\vec{\nabla} \times(\vec{V} \times \vec{B})+\eta \nabla^{2} \vec{B}
$$

Where $\eta$-is coefficient of magnetic diffusivity. $\overrightarrow{\mathrm{V}}$-is the local velocity of the conductivity fluid motion in the liquid core.

It is possibility that the motions in the liquid core are divided two components, mean motions and turbulent motions. Velocity of the mean motions is steadily and its L-scale is long. The turbulent motions' $l$-scale is small as $(L>>l)$. Velocity of the turbulent motions is changed quickly and it is with fluctuation. These are written in the following form, on the $\mathrm{Eq}(2)$.

$$
\left.\begin{array}{rl}
\vec{V} & =\overrightarrow{V_{0}}+\vec{V}=\text { mean }+ \text { fluctuations } \\
\vec{B} & =\overrightarrow{B_{0}}+\vec{b}=\text { mean + fluctuations }
\end{array}\right\}
$$

After the $\mathrm{Eq}(2)$ is replaced into the Eq (1), the Eq (1) should be averaged because the secular variation observed on the Earth's surface is with long time scale and the fluctuation effect is very small in it. The Eq (1) is averaged by the $\mathrm{Eq}(3)$. The $\mathrm{Eq}(3)$ is named Reynolds rules for averages [6],[10].

$$
\left.\begin{array}{c}
\left\langle\overrightarrow{\mathrm{V}_{0}}\right\rangle=\overrightarrow{\mathrm{V}_{0}},\langle\overrightarrow{\mathrm{v}}\rangle=0 \\
\langle\overrightarrow{\mathrm{V}}\rangle=\left\langle\overrightarrow{\mathrm{V}_{0}}+\overrightarrow{\mathrm{V}}\right\rangle=\left\langle\overrightarrow{\mathrm{V}_{0}}\right\rangle+\langle\overrightarrow{\mathrm{V}}\rangle=\overrightarrow{\mathrm{V}_{0}} \\
\left\langle\overrightarrow{\mathrm{B}_{0}}\right\rangle=\overrightarrow{\mathrm{B}_{0}},\langle\overrightarrow{\mathrm{b}}\rangle=0 \\
\langle\overrightarrow{\mathrm{B}}\rangle=\left\langle\overrightarrow{\mathrm{B}_{0}}+\overrightarrow{\mathrm{b}}\right\rangle=\left\langle\overrightarrow{\mathrm{B}_{0}}\right\rangle+\langle\overrightarrow{\mathrm{b}}\rangle=\overrightarrow{\mathrm{B}_{0}}
\end{array}\right\}
$$

$\langle\cdots\rangle$ statistical averaging, $(\approx$ volume or time averaging).

$\left\langle\frac{\mathrm{d} \overrightarrow{0_{0}}}{\mathrm{dt}}+\frac{\mathrm{d} \overrightarrow{\mathrm{b}}}{\mathrm{dt}}\right\rangle=\vec{\nabla} \times\left\langle\left(\overrightarrow{\mathrm{V}_{0}}+\overrightarrow{\mathrm{v}}\right) \times\left(\overrightarrow{\mathrm{B}_{0}}+\overrightarrow{\mathrm{b}}\right)\right\rangle+\eta \nabla^{2}\left\langle\overrightarrow{\mathrm{B}_{0}}+\overrightarrow{\mathrm{b}}\right\rangle$

After the Eq(4) is averaged by Reynolds rules for averages, it is rewritten on the $\mathrm{Eq}(5)$.

$\frac{d \overrightarrow{\mathrm{B}_{0}}}{\mathrm{dt}}=\vec{\nabla} \times\left(\overrightarrow{\mathrm{V}_{0}} \times \overrightarrow{\mathrm{B}_{0}}\right)+\vec{\nabla} \times\langle\overrightarrow{\mathrm{v}} \times \overrightarrow{\mathrm{b}}\rangle+\eta \nabla^{2} \overrightarrow{\mathrm{B}_{0}}$

The $\operatorname{Eq}(5)$ is named the averaged induction equation. $\vec{\nabla} \times\langle\overrightarrow{\mathrm{v}} \times \overrightarrow{\mathrm{b}}\rangle$-this term on the $\operatorname{Eq}(5)$ expresses the effect of turbulence in the mean magnetic field. The Eq (6) is named the mean electromotive force.

$$
\vec{\varepsilon}=\langle\vec{v} \times \vec{b}\rangle
$$

There is a linear relation between the mean electromotive force $\vec{\varepsilon}$ and the mean magnetic field $\overrightarrow{\mathrm{B}_{0}}$.

It can be shown on the $\operatorname{Eq}(7)$. The $\operatorname{Eq}(7)$ is named Taylor's expansion [10],[6].

$$
\left.\begin{array}{c}
\vec{\varepsilon} \approx \alpha \overrightarrow{\mathrm{B}_{0}}-\beta \vec{\nabla} \times \overrightarrow{\mathrm{B}_{0}}+\cdots \\
\alpha=\frac{1}{3}\langle\overrightarrow{\mathrm{v}} \cdot(\vec{\nabla} \times \overrightarrow{\mathrm{v}})\rangle \tau \\
\beta=\frac{1}{3} \mathrm{v}^{2} \tau
\end{array}\right\}
$$

Where $\alpha$ is helical effects' coefficient of fluent motion in the liquid core. $\beta$-is coefficient for the turbulent diffusivity.

After the $\mathrm{Eq}(7)$ is replaced into the $\mathrm{Eq}(5)$, it is rewritten on the $\mathrm{Eq}(8)$. The $\mathrm{Eq}(8)$ is the governing equation to study the secular variation of the main geomagnetic field.

$$
\frac{\mathrm{d} \overrightarrow{\mathrm{B}_{0}}}{\mathrm{dt}}=\vec{\nabla} \times\left(\overrightarrow{\mathrm{V}_{0}} \times \overrightarrow{\mathrm{B}_{0}}\right)+\vec{\nabla} \times \alpha \overrightarrow{\mathrm{B}_{0}}+(\eta+\beta) \nabla^{2} \overrightarrow{\mathrm{B}_{0}}(8)
$$

After $\left(\vec{\nabla} \times\left(\overrightarrow{V_{0}} \times \overrightarrow{B_{0}}\right)=-\left(\overrightarrow{V_{0}} \cdot \vec{\nabla}\right) \overrightarrow{B_{0}}+\left(\overrightarrow{B_{0}} \cdot \vec{\nabla}\right) \overrightarrow{V_{0}}-\overrightarrow{B_{0}}\left(\vec{\nabla} \cdot \overrightarrow{V_{0}}\right)\right.$ )-this expansion is replaced into the $\mathrm{Eq}(8)$, it is rewritten on the $\operatorname{Eq}(9)$.

$\frac{a \overline{B_{0}}}{d t}=-\left(\overrightarrow{V_{0}} \cdot \vec{\nabla}\right) \overrightarrow{B_{0}}+\left(\overrightarrow{B_{0}} \cdot \vec{\nabla}\right) \overrightarrow{V_{0}}-\overrightarrow{\mathrm{B}_{0}}\left(\vec{\nabla} \cdot \overrightarrow{V_{0}}\right)+\vec{\nabla} \times\left(\alpha \overrightarrow{\mathrm{B}_{0}}\right)+(\eta+\beta) \nabla^{2}\left(\overrightarrow{\mathrm{B}_{0}}\right)$

Where $\left(\overrightarrow{V_{0}} \cdot \vec{\nabla}\right) \overrightarrow{\mathrm{B}_{0}}$ is advection's term. $\left(\overrightarrow{\mathrm{B}_{0}} \cdot \vec{\nabla}\right) \overrightarrow{V_{0}}$ is stretching's term. $\overrightarrow{\mathrm{B}_{0}}\left(\vec{\nabla} \cdot \overrightarrow{V_{0}}\right)$ is compression's term. $(\eta+\beta) \nabla^{2}\left(\overrightarrow{B_{0}}\right)$ is diffusion or decay's term. $\overrightarrow{V_{0}}$ - is the mean velocity of the conductivity fluid motion in the liquid core.

It is possibility that the $\mathrm{Eq}(9)$ is rewritten on the form $\mathrm{Eq}(10)$.

$$
\frac{d \overrightarrow{\mathrm{B}_{0}}}{d t}+\left(\overrightarrow{V_{0}} \cdot \vec{\nabla}\right) \overrightarrow{\mathrm{B}_{0}}=\left(\overrightarrow{\mathrm{B}_{0}} \cdot \vec{\nabla}\right) \overline{V_{0}}-\overrightarrow{\mathrm{B}_{0}}\left(\vec{\nabla} \cdot \overrightarrow{V_{0}}\right)+\vec{\nabla} \times\left(\alpha \overrightarrow{\mathrm{B}_{0}}\right)+(\eta+\beta) \nabla^{2}\left(\overrightarrow{\mathrm{B}_{0}}\right)
$$

Generally, $\overrightarrow{V_{0}}$-is steady in the liquid core and its local variation is very small. Thus, 
$\left(\overrightarrow{\mathrm{B}_{0}} \cdot \vec{\nabla}\right) \overrightarrow{V_{0}}$ and $\overrightarrow{\mathrm{B}_{0}}\left(\vec{\nabla} \cdot \overrightarrow{V_{0}}\right)$ terms' effects are small on the $\mathrm{Eq}(10)$ for the Earth's liquid core.

As reword its, the stretching and the compression exist very small or don't exist in the conductivity fluid motion in the Earth's liquid core.

$\vec{\nabla} \times\left(\alpha \overrightarrow{\mathrm{B}_{0}}\right)$ and $\beta \nabla^{2}\left(\overrightarrow{\mathrm{B}_{0}}\right)$-terms on the $\operatorname{Eq}(10)$ are turbulent effects in the liquid core. The $\alpha$ and $\beta$ are with small scale. The $\tau$-correlation time in the $\alpha$ and $\beta$ on the $\operatorname{Eq}(7)$ is also small. Therefore, the terms' effects are observed probably about one point or one observatory on the Earth's surface. But our distribution functions of $\mathrm{Bx}, \mathrm{By}$ and $\mathrm{Bz}$ envelop continents such as the Europe and the north America. Thus these terms' effects with $\alpha$ and $\beta$ are decreased in the distribution functions of $\mathrm{Bx}, \mathrm{By}$ and $\mathrm{Bz}$ how they are averaged by the large areas of the Europe and the north America. Therefore these terms with $\alpha$ and $\beta$ cause very small in the same kind equation with the $\operatorname{Eq}(10)$ what will be written on the surface of the Earth or in the mantle.

The diffusion's term $\eta \nabla^{2}\left(\overrightarrow{\mathrm{B}_{0}}\right)$ on the $\operatorname{Eq}(1)$ is 500-1000 times weaker than $\vec{\nabla} \times(\vec{V} \times \vec{B})$ the induction's term on the $\operatorname{Eq}(1)$ by the magnetic Reynold's ratio in the Earth's liquid core. Thus $\eta \nabla^{2}\left(\overrightarrow{\mathrm{B}_{0}}\right)$-this term's effect is very small in the $\mathrm{Eq}(10)$.

Above the $\mathrm{Eq}(10)$ is only valid in the liquid core. But it is looked from the $\mathrm{Eq}(10)$ that the main factor is advection's term in secular variation of MGF one point on the Earth's surface.

We can write an equation of the same kind with the $\mathrm{Eq}(10)$ on the Earth's surface. The equation is written in the following form, on the $\mathrm{Eq}(11)$.

$$
\frac{d \vec{B}}{d t}+\left(\overrightarrow{v_{0}^{\prime}} \cdot \vec{\nabla}\right) \vec{B}=\vec{R}(r, t)
$$

Where $\mathrm{r}$ - denotes the positional vector and $\overrightarrow{v_{0}^{\prime}}$ is the drift's velocity of the field on the Earth's surface or in the mantle. $\vec{R}(r, t)$-is the residual field after the variation due to the westward drift has been subtracted from the observed time-variation, and it enables us to estimate the field variation actually taking place in the Earth's liquid core as described by the right hand side of the Eq(10). But these

terms'effects in residual field of the $\mathrm{Eq}(11)$ are observed weaker than terms of the Eq(10) on the Earth's surface. Because these effects are decreased by mantle.

If we have knowledge on the rate of change $d \vec{B}$

$\frac{d \vec{B}}{d t}$ and the field distribution $\vec{B}$, we are able to adjust the velocity $\overrightarrow{v_{0}^{\prime}}$ so as to minimize the residual field variation $\vec{R}(r, t)$.

We will estimate the velocity $\overrightarrow{v_{0}^{\prime}}$ from the least square method which integrates the square of residual field $\vec{R}(r, t)$ by intervals of time and space. It is written in the following form, on the Eq (12).

$$
\Phi=\int[\vec{R}(r, t)]^{2} d s
$$

Where ds- is intervals of space and time. $\vec{R}(r, t)$ -is residual field.

We can estimate the velocity $\overrightarrow{v_{0}^{\prime}}$ from the minimum condition of the functional on the Eq (12).

\section{Methods of the estimation}

On the North America and the Europe in (1991-2006), we possess the distribution functions of MGF by form on the $\mathrm{Eq}(13)$ and the $\mathrm{Eq}(14)$ what depended on the space and time. The Eq(13) and the Eq(14) are also written in coordinate system NED.

$$
\left.\begin{array}{l}
\operatorname{Bx}(\theta, \varphi)=\mathrm{C}_{00}+\mathrm{C}_{01} \varphi+\mathrm{C}_{02} \varphi^{2}+\mathrm{C}_{03} \varphi^{3}+\mathrm{C}_{10} \theta+\mathrm{C}_{11} \theta \varphi+\mathrm{C}_{12} \theta \varphi^{2}+\mathrm{C}_{20} \theta^{2}+\mathrm{C}_{21} \varphi \theta^{2}+\mathrm{C}_{30} \theta^{3} \\
\operatorname{By}(\theta, \varphi)=\mathrm{A}_{00}+\mathrm{A}_{01} \varphi+\mathrm{A}_{02} \varphi^{2}+\mathrm{A}_{03} \varphi^{3}+\mathrm{A}_{10} \theta+\mathrm{A}_{11} \theta \varphi+\mathrm{A}_{12} \theta \varphi^{2}+\mathrm{A}_{20} \theta^{2}+\mathrm{A}_{21} \varphi \theta^{2}+\mathrm{A}_{30} \theta^{3} \\
\operatorname{Bz}(\theta, \varphi)=\mathrm{K}_{00}+\mathrm{K}_{01} \varphi+\mathrm{K}_{02} \varphi^{2}+\mathrm{K}_{03} \varphi^{3}+\mathrm{K}_{10} \theta+\mathrm{K}_{11} \theta \varphi+\mathrm{K}_{12} \theta \varphi^{2}+\mathrm{K}_{20} \theta^{2}+\mathrm{K}_{21} \varphi \theta^{2}+\mathrm{K}_{30} \theta^{3}
\end{array}\right\}
$$


Time variations in polynomial coefficients C, A, K, D, Q and $\mathrm{H}$ on the Eq(13-14) are defined above the article named the distribution function of MGF on space and time on the North America and the Europe. Thus we used in this paper these time variations in polynomial coefficients from the above article.

The coordinate system NED(X-north, Yeast, Z-down) is used in observatories of intermagnet.org. Westward drift's velocity is detected in the coordinate system Earth-Centered Earth-Fixed (ECEF). The (ECEF) or conventional terrestrial coordinate system rotates with the Earth and has its origin at the center of the Earth. The $\mathrm{X}$-axis passes through the equator at the prime meridian.The $\mathrm{Z}$-axis passes through the North Pole. The Y-axis can be determined by the right-hand rule to be passing through the equator at 90-degree longitude.

Thus the above distribution functions of geomagnetic field in coordinate system NED on the $\mathrm{Eq}(13)$ and the $\mathrm{Eq}(14)$ are transformed to (ECEF) by the Eq(15) on the Earth's surface.

$$
\overrightarrow{\mathrm{B}}_{\mathrm{e}}=\hat{I} \vec{B}
$$

Where $\overrightarrow{\mathrm{B}_{\mathrm{e}}}$-is vector in coordinate system (ECEF). $\vec{B}$-is vector in coordinate system (NED).

$\hat{I}$-Inversion tensor and its determination on the $\mathrm{Eq}(15)$ are rewritten on the $\mathrm{Eq}(16)$.

$|\hat{I}|=\left|\begin{array}{ccc}-\sin (\theta) \cos (\varphi) & -\sin (\varphi) & -\cos (\theta) \cos (\varphi) \\ -\sin (\theta) \sin (\varphi) & \cos (\varphi) & -\cos (\theta) \sin (\varphi) \\ \cos (\theta) & 0 & -\sin (\theta)\end{array}\right|=1$

Now, the residual field on the $\mathrm{Eq}(11)$ is rewritten on the $\mathrm{Eq}(17)$. The $\mathrm{Eq}(15)$ is also replaced into the $\operatorname{Eq}(17)$.

$$
\vec{R}_{\mathrm{e}}(r, t)=\frac{d \overrightarrow{\mathrm{B}}_{\mathrm{e}}}{d t}+\left(\overrightarrow{v_{0}^{\prime}} \cdot \vec{\nabla}_{\mathrm{e}}\right) \overrightarrow{\mathrm{B}}_{\mathrm{e}}=\frac{d \hat{\imath} \vec{B}}{d t}+\left(\overrightarrow{v_{0}^{\prime}} \cdot \vec{\nabla}_{\mathrm{e}}\right) \hat{I} \vec{B}
$$

We must define the quadrat of the residual field on the $\mathrm{Eq}(17)$. It is written on the $\operatorname{Eq}(18)$.

$\left[\vec{R}_{\mathrm{e}}(r, t)\right]^{2}=\hat{I}^{2} \frac{d \vec{b}}{d t} \cdot \frac{d \vec{b}}{d t}+2\left(\overrightarrow{v_{0}} \cdot \vec{\nabla}_{\mathrm{e}}\right) \hat{I}^{2}\left(\vec{B} \cdot \frac{d \vec{b}}{d t}\right)+\left(\overrightarrow{v_{0}^{\prime}} \cdot \vec{\nabla}_{\mathrm{e}}\right)^{2} \hat{I}^{2}(\overrightarrow{\mathrm{B}} \cdot \overrightarrow{\mathrm{B}})$.

Where $\hat{I}^{2}-$ is inversion tensor's quadrat. It is $\hat{I}^{2}=1 . \vec{\nabla}_{\mathrm{e}}$ is Nable's operator in (ECEF).

It is possibility that the $\operatorname{Eq}(18)$ is rewritten on the Eq(19). Because any vector's quadrat is not varied by the inversion operation or the rotation of coordinate system.

$$
\left[\vec{R}_{\mathrm{e}}(r, t)\right]^{2}=\left[\frac{d \vec{B}}{d t}+\left(\overrightarrow{v_{0}^{\prime}} \cdot \vec{\nabla}_{\mathrm{e}}\right) \vec{B}\right]^{2}
$$

Now, we are able to adjust the functional denoted via the $\operatorname{Eq}(20)$.

$$
\Phi=\int\left[\vec{R}_{\mathrm{e}}(r, t)\right]^{2} d s
$$

Components of residual field on the $\mathrm{Eq}(19)$ are denoted as the $\operatorname{Eq}(21)$.

$\left.\begin{array}{l}R x(r, t)=\vec{\imath} \frac{d B x}{d t}+\vec{\imath}\left[v_{0 x}^{\prime} \frac{d \mathrm{Bx}}{d x}+v_{0 y}^{\prime} \frac{d \mathrm{Bx}}{d y}+v_{0 z}^{\prime} \frac{d \mathrm{Bx}}{d z}\right] \\ R y(r, t)=\vec{\jmath} \frac{d B y}{d t}+\vec{\jmath}\left[v_{0 x}^{\prime} \frac{d \mathrm{By}}{d x}+v_{0 y}^{\prime} \frac{d \mathrm{By}}{d y}+v_{0 z}^{\prime} \frac{d \mathrm{By}}{d z}\right] \\ R z(r, t)=\vec{k} \frac{d B y}{d t}+\vec{k}\left[v_{0 x}^{\prime} \frac{d \mathrm{Bz}}{d x}+v_{0 y}^{\prime} \frac{d \mathrm{Bz}}{d y}+v_{0 z}^{\prime} \frac{d \mathrm{Bz}}{d z}\right]\end{array}\right\}$

When the study of westward drift is done on the North America, the $\mathrm{Eq}(13)$ are replaced into the $\mathrm{Eq}(21)$.

In the coordinate system (ECEF), the components of drift's velocity on the $\operatorname{Eq}(21)$ are also denoted by the $\mathrm{Eq}(22)$.

$$
\left.\begin{array}{c}
v_{0 x}^{\prime}=-r \sin \theta \cdot \cos \varphi \cdot \dot{\theta}-r \cdot \cos \theta \cdot \sin \varphi \cdot \omega \\
v_{0 y}^{\prime}=-r \sin \theta \cdot \sin \varphi \cdot \dot{\theta}+r \cdot \cos \theta \cdot \cos \varphi \cdot \omega \\
v_{0 z}^{\prime}=r \cdot \cos \theta \cdot \dot{\theta}
\end{array}\right\}
$$

If the $\mathrm{Eq}(21)$ and the $\mathrm{Eq}(22)$ replace into the functional on the $\mathrm{Eq}(20)$, it can be rewritten on the $\operatorname{Eq}(23)$.

$$
\Phi_{\mathrm{am}}(\omega, \dot{\theta})=\int_{1991}^{2006}\left[\int_{0}^{R} \int_{\frac{\pi}{9}}^{\frac{4 \pi}{9}} \int_{\frac{10 \pi}{9}}^{\frac{16 \pi}{9}}\left[(F x)^{2}+(F y)^{2}+(F z)^{2}\right] r^{2} \cos \theta d \varphi d \theta d r\right] d t
$$


Where $F x, F y$ and $F z$ are written in the following on Eq. (24)

$$
\left.\begin{array}{l}
F x=a 1+b 1 \cdot \omega+c 1 \cdot \dot{\theta} \\
F y=a 2+b 2 \cdot \omega+c 2 \cdot \dot{\theta} \\
F z=a 3+b 3 \cdot \omega+c 3 \cdot \dot{\theta}
\end{array}\right\}
$$

Where a1, a2, a3, b1, b2, b3, c1, c2 and c3 are written in the following expressions (25).

$$
\left.\begin{array}{c}
a 1=\frac{d \mathrm{~B} x}{d t}, a 2=\frac{d \mathrm{By}}{d t}, a 3=\frac{d \mathrm{Bz}}{d t} \\
b 1=\frac{d \mathrm{Bx}}{d y} \mathrm{r} \cos \theta \cos \varphi-\frac{d \mathrm{Bx}}{d x} \mathrm{r} \cos \theta \sin \varphi \\
b 2=\frac{d \mathrm{By}}{d y} \mathrm{r} \cos \theta \cos \varphi-\frac{d \mathrm{By}}{d x} \mathrm{r} \cos \theta \sin \varphi \\
b 3=\frac{d \mathrm{Bz}}{d y} \mathrm{r} \cos \theta \cos \varphi-\frac{d \mathrm{Bz}}{d x} \mathrm{r} \cos \theta \sin \varphi \\
c 1=\frac{d \mathrm{Bx}}{d z} \mathrm{r} \cos \theta-\frac{d \mathrm{Bx}}{d y} \mathrm{r} \sin \theta \sin \varphi-\frac{d \mathrm{Bx}}{d x} \mathrm{r} \sin \theta \cos \varphi \\
c 2=\frac{d \mathrm{By}}{d z} \mathrm{r} \cos \theta-\frac{d \mathrm{By}}{d y} \mathrm{r} \sin \theta \sin \varphi-\frac{d \mathrm{By}}{d x} \mathrm{r} \sin \theta \cos \varphi \\
c 3=\frac{d \mathrm{Bz}}{d z} \mathrm{r} \cos \theta-\frac{d \mathrm{Bz}}{d y} \mathrm{r} \sin \theta \sin \varphi-\frac{d \mathrm{Bz}}{d x} \mathrm{r} \sin \theta \cos \varphi
\end{array}\right\}
$$

We have detected the latitudinal and longitudinal drifts from the minimum condition of the functional on the $\mathrm{Eq}(23)$.

\section{The results and discussion}

After the functional on the Eq.(23) is integrated by intervals of space and time written on the Eq.(23), it is rewritten in the following on the Eq. (26).

$\Phi_{\mathrm{am}}(\omega, \dot{\theta})=R^{3}\left(m_{1} \dot{\theta}+\mathrm{m}_{2} \omega+\mathrm{m}_{3} \dot{\theta} \omega+\mathrm{m}_{4} \dot{\theta}^{2}+\mathrm{m}_{5}\left(\omega^{2}\right)\right.$

Where $\mathrm{m}_{1}, \mathrm{~m}_{2}, \mathrm{~m}_{3}, \mathrm{~m}_{4}$ and $\mathrm{m}_{5}$ are coefficients. And the coefficients are calculated on the
$\operatorname{Eq}(27)$.

$$
\left.\begin{array}{c}
\mathrm{m}_{1}=-98084362.413, \mathrm{~m}_{2}=5746585.905 \\
\mathrm{~m}_{3}=10852928483.565, \mathrm{~m}_{4}=23151710149.065 \\
\mathrm{~m}_{5}=4602283136.647
\end{array}\right\}
$$

The minimum condition of the functional on the $\mathrm{Eq}(26)$ is written on the $\mathrm{Eq}(28)$.

$$
\left.\begin{array}{l}
\frac{\partial \Phi(\omega, \dot{\theta})}{\partial \dot{\theta}}=0 \\
\frac{\partial \Phi(\omega, \dot{\theta})}{\partial \omega}=0
\end{array}\right\}
$$

The expression on the $\mathrm{Eq}(28)$ can also be rewritten as the following linear equations system, on the $\mathrm{Eq}(29)$.

$$
\left.\begin{array}{c}
\mathrm{m}_{1}+\mathrm{m}_{3} \omega+2 \mathrm{~m}_{4} \dot{\theta}=0 \\
\mathrm{~m}_{2}+\mathrm{m}_{3} \dot{\theta}+2 \mathrm{~m}_{5} \omega=0
\end{array}\right\}
$$

We can solve the linear equations system on the $\operatorname{Eq}(29)$. The solutions of the $\operatorname{Eq}(29)$ are written on the $\operatorname{Eq}(30)$.

$$
\left.\begin{array}{c}
\omega=-0.247 \frac{\text { degree }}{\text { year }} \\
\dot{\theta}=0.179 \frac{\text { degree }}{\text { year }}
\end{array}\right\}
$$

By the results of this investigation, longitudinal and latitudinal drifts exist on the North America in (1991-2006).

When the study of westward drift is done on the Europe, the $\mathrm{Eq}(14)$ are replaced into the functional on the $\mathrm{Eq}(20)$. Moreover, intervals of the integral on the $\mathrm{Eq}(23)$ are changed as the $\mathrm{Eq}(31)$.

$$
\Phi_{\mathrm{eu}}(\omega, \dot{\theta})=\int_{1991}^{2006}\left[\int_{0}^{R} \int_{\frac{\pi}{6}}^{\frac{4 \pi}{9}} \int_{\frac{-\pi}{18}}^{\frac{2 \pi}{9}}\left[(F x)^{2}+(F y)^{2}+(F z)^{2}\right] r^{2} \cos \theta d \varphi d \theta d r\right] d t
$$

After the functional on the $\mathrm{Eq}(31)$ is integrated by the above intervals, it is rewritten on the $\operatorname{Eq}(32)$.

$$
\Phi_{\mathrm{eu}}(\omega, \dot{\theta})=R^{3}\left(n_{1} \dot{\theta}+\mathrm{n}_{2} \omega+\mathrm{n}_{3} \dot{\theta} \omega+\mathrm{n}_{4} \dot{\theta}^{2}+\mathrm{n}_{5} \omega^{2}\right)
$$

Where $\mathrm{n}_{1}, \mathrm{n}_{2}, \mathrm{n}_{3}, \mathrm{n}_{4}$ and $\mathrm{n}_{5}$ are coefficients. And the coefficients' values are calculated on the $\mathrm{Eq}(33)$. 
$\mathrm{n}_{1}=2616757.101, \mathrm{n}_{2}=1042972.699$

$\mathrm{n}_{3}=642390651.089, \mathrm{n}_{4}=5622584744.759$

$\mathrm{n}_{5}=124977085.644$

The following linear equations system on the $\mathrm{Eq}(34)$ is written from the minimum condition of the functional on the $\operatorname{Eq}(32)$.

$$
\left.\begin{array}{c}
\mathrm{n}_{1}+\mathrm{n}_{3} \omega+2 \mathrm{n}_{4} \dot{\theta}=0 \\
\mathrm{n}_{2}+\mathrm{n}_{3} \dot{\theta}+2 \mathrm{n}_{5} \omega=0
\end{array}\right\}
$$

The solutions of the $\operatorname{Eq}(34)$ are written on the $\mathrm{Eq}(35)$.

$$
\left.\begin{array}{c}
\omega=-0.240 \frac{\text { degree }}{\text { year }} \\
\dot{\theta}=3.805 \cdot 10^{-4} \frac{\text { degree }}{\text { year }}
\end{array}\right\}
$$

We can define the westward drift by this method on the part of Earth's surface where the MGF's distribution functions of space and time are known clearly. If we can detect clearly the MGF's distribution functions of space and time on the continents, islands and peninsulas, we will define the westward drifts by this method on these areas such as continents of Africa and Australia, islands of England and Japan, peninsulas of India, Arabic and Korea.

\section{Conclusion}

The advection's term $-\left(\overrightarrow{V_{0}} \cdot \vec{\nabla}\right) \overrightarrow{\mathrm{B}_{0}}$ on the $\mathrm{Eq}(9)$ is the main factor in MGF's the variation of time or the secular variation. If it is reworded, the mean geomagnetic field's induction equation on the $\mathrm{Eq}(10)$ for the Earth's liquid core is depended very weakly on the terms of right hand from the equal. These are the theoretical basic of this method to estimate the westward drift.

It is possibility that the westward drift is estimated by this method on the little area of Earth's surface which the geomagnetic field's distribution function of space and time is known clearly.

In this paper, we detected the mean geomagnetic field's distribution function of space and time by data of observatories

located on the North America and the Europe. And the westward drifts are estimated in the following forms.

Longitudinal drift $\omega=-0.247 \frac{\text { degree }}{\text { vear }} \$$ and latitudinal drift $\dot{\theta}=0.179 \frac{\text { degree }}{\text { vear }}$ existed on the North America in (1991-2006). And longitudinal drift $\omega=-0.240 \frac{\text { degree }}{\text { year }}$ is defined by the above method on the Europe in (1991-2006). The latitudinal drift doesn't exist on the Europe in (1991-2006).

These drifts (1991-2006) are approximation with investigation's results by other methods.

\section{References}

[1] Takesi Yukutake, The westward drift of the magnetic field of the Earth, Bulletin of the earthquake research Institute Vol.40 (1962) pp (1-65)

[2] Takesi Yukutake and Hiroko Tachinaka, The westward drift of the geomagnetic secular variation, Bulletin of the earthquake research Institute Vol.46 (1968) pp (1075-1102)

[3] Bullard, E. C., Freedman, C., Gellman, H. et al., The westward drift of the Earth's magnetic field, Phil. Trans. R. Soc. Lond., 1950, A243: 67-92.

[4] Zigang Wei and Wenyao Xu, Differential rotation of geomagnetic field, Chinese Science Bulletin Volume 48, Number 24, 2739-2742, DOI: 10.1007/BF02901767

[5] Mathieu Dumberry, Christopher C. Finlay, Eastward and westward drift of the Earth's magnetic field for the last three millennia, Earth and Planetary Science Letters 254 (2007) pp (146-157)

[6] Anvar Shukurov, Dynamo Theory and Galaxies, in Newcastle university press in 2001.

[7] Herndon, J. M., Nuclear georeactor generation of earth's geomagnetic field. CURRENT SCIENCE 93, 1485-1487, 2007

[8] Herndon, J. M., Nature of planetary matter and magnetic field generation in the solar system CURRENT SCIENCE ( VOL. 96, NO. 8, 25 APRIL 2009)

[9] Herndon, J. M., Examining the Overlooked Implications of Natural Nuclear Reactors, Eos, Transactions, American Geophysical Union, Vol. 79, No. 38, Pages 451,456, September 2,1998

[10] Radler.K.H, Mean-field dynamo theory: early ideas and today's problems, Astrophysical Institute Potsdam, An der Sternwarte 16, D-14482 Potsdam, Germany. 